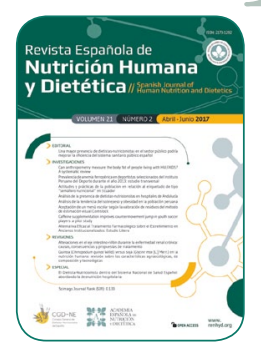

\title{
ARTÍCULO DE INVESTIGACIÓN \\ Prevalencia de anemia ferropénica en deportistas seleccionados del Instituto Peruano del Deporte durante el año 2013: estudio transversal
}

\author{
Alexandra Rivera Ameri ${ }^{a}$, Valeria Quiroz Acurio ${ }^{a, *}$, Kevyn Arias Montano ${ }^{a}$ \\ aniversidad Peruana de Ciencias Aplicadas, Perú. \\ *valeria_93_2@hotmail.com
}

Recibido el 13 de septiembre de 2016; aceptado el 20 de febrero de 2017; publicado el 9 de mayo de 2017.

PALABRAS CLAVE
Anemia Ferropénica;
Hemoglobina;
Sexo;
Atletas;
Deportes.

Prevalencia de anemia ferropénica en deportistas seleccionados del Instituto Peruano del Deporte durante el año 2013: estudio transversal

\section{RESUMEN}

Introducción: El objetivo de este trabajo fue determinar la prevalencia de anemia ferropénica según el componente dinámico y estático, identificando de esta manera los deportes con mayor prevalencia de anemia, y determinar la asociación entre el sexo, la edad y anemia.

Material y Métodos: Estudio observacional de tipo corte transversal analítico. Se utilizó la base de datos de la Dirección Nacional de Servicios Biomédicos del Instituto Peruano del Deporte, en el período de enero a diciembre de 2013. De un universo de 1833 deportistas, se seleccionaron aquellos entre 18 y 35 años de edad. Las variables numéricas se evaluaron usando la prueba de T de Student o la prueba de Mann-Whitney dependiendo del estado de normalidad de las variables. Las variables categóricas fueron evaluadas usando $\chi^{2}$. Razones de prevalencias crudas y ajustadas fueron calculadas usando regresión de Poisson con varianza robusta, para evaluar la asociación entre sexo y anemia.

Resultados: Se analizaron 633 deportistas, siendo en su mayoría varones (68,4\%). Las variables que se incluyeron en el modelo bivariado fueron sexo, edad deportiva y suplementación con hierro, las cuales fueron también incluidas en el modelo multivariado, encontrándose asociación entre anemia y edad deportiva $(p=0,01)$. No se encontró asociación entre sexo y anemia

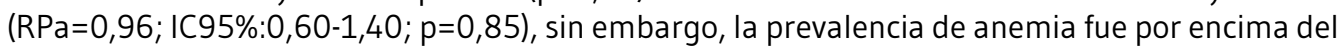
$15 \%$ (17,9\% hombres y $16,8 \%$ mujeres).

Conclusiones: Se halló mayor prevalencia de anemia ferropénica en los deportes de alto componente estático y dinámico. No se encontró asociación entre anemia ferropénica y sexo en deportistas seleccionados del Instituto Peruano del Deporte en el año 2013. 


\section{KEYWORDS}

Anemia, Iron-Deficiency;

Hemoglobins;

Sex;

Athletes;

Sports.

Prevalence of iron-deficiency anemia in selected athletes from the Peruvian Institute of Sport during 2013: cross-sectional study

\section{ABSTRACT}

Introduction: The aims of this study were to determine the iron-deficiency anemia prevalence per dynamic and static component and thus identify sports with the highest prevalence of irondeficiency anemia, and to determine the association between sports, sex, age and iron-deficiency anemia.

Material and Methods: Observational study and cross-sectional analytic. The database of the National Biomedical Services Directorate of the Peruvian Institute of Sport was used for the period from January to December 2013. Athletes aged 18-35 were selected from a total universe of 1833 athletes. Numerical variables were evaluated using the Student's T-test or the Mann-Whitney U-test, depending on the normality of the variables. Categorical variables were evaluated using the $\chi^{2}$ test. Crude and adjusted Prevalence Ratios were calculated using the Poisson regression with robust error variance, to assess the association between sex and iron-deficiency anemia.

Results: 633 athletes were analyzed, being mostly male (68.4\%). Variables included in the bivariate model were sex, sports age and iron supplementation, which were also included in the multivariate model, and an association between iron-deficiency anemia and sports age $(p=0.01)$ was found. No association between sex and iron-deficiency anemia was found ( $\mathrm{RPa}=0.96$; $95 \% \mathrm{Cl}: 0.60-1.40 ; p=0.85)$. However, the prevalence of iron-deficiency anemia was above $15 \%$ (17.9\% men and $16.8 \%$ women). The main result was that there is no significant difference when comparing the prevalence of iron-deficiency anemia between men and women (17.9\% and $16.8 \%$ ).

Conclusions: Higher prevalence of iron-deficiency anemia was found in sports with high static and dynamic component. There is not association between iron-deficiency anemia and sex in athletes from the Peruvian Institute of Sport in 2013.

\section{CITA}

Rivera Ameri A, Quiroz Acurio V, Arias Montano K. Prevalencia de anemia ferropénica en deportistas seleccionados del Instituto Peruano del Deporte durante el año 2013: estudio transversal. Rev Esp Nutr Hum Diet. 2017; 21(2): 112-20. doi: 10.14306/renhyd.21.2.280

\section{INTRODUCCIÓN}

La anemia se define como una disminución del número de glóbulos rojos en sangre, limitando el transporte de oxígeno; lo cual implica que no se satisfagan las necesidades del organismo ${ }^{1}$. Los principales tipos de anemia son la anemia ferropénica (por una deficiencia de hierro), la anemia microcítica, macrocítica y megaloblástica, entre otras²; aunque la anemia más frecuente y la primera causa de visita hematológica es la anemia ferropénica. La deficiencia de las reservas de este micronutriente en ambos sexos tiene consecuencias no sólo hematológicas sino también sistémicas, ya que influye sobre la energía corporal, capacidad de trabajo, desarrollo en diferentes etapas de vida, funciones inmunológicas, función cognitiva, entre otras. Según la Organización Mundial de la Salud (OMS), se puede diagnosticar anemia cuando el rango de hemoglobina es $<13 \mathrm{mg} / \mathrm{dL}$ para hombres y $<12 \mathrm{mg} / \mathrm{dL}$ para mujeres ${ }^{1}$. Asimismo, Perú tiene la prevalencia más alta de anemia de toda Sudamérica y la prevalencia de la misma según la encuesta ENDES 2014 es de $21,6 \%$ en mujeres en edad fértil y de $35,6 \%$ en niños menores de 5 años ${ }^{3}$.

La prevalencia de anemia es mayor en mujeres que en hombres debido a que existe una mayor predisposición a presentar menores niveles de hemoglobina ya que, a nivel fisiológico, éstas presentan un volumen pulmonar un $10 \%$ menor que el género masculino y menor concentración plasmática de hemoglobina, por ende, menor capacidad de consumo máximo de oxígeno?

Por otro lado, diversos estudios han mostrado que los profesionales dedicados al deporte pueden presentar una mayor prevalencia de anemia que la población general ${ }^{4-9}$ debido 
a la intensidad del entrenamiento, al consumo de fármacos y a la deficiencia de ingesta de alimentos ricos en este nutriente en la dieta 4 . Además, un estudio previo mostró que aquellos deportistas que practican actividades de resistencia o deportes en equipo de alto impacto tienen una mayor prevalencia de anemia ${ }^{10}$. La anemia en el deportista profesional es de suma importancia debido que limita la capacidad del atleta para realizar distintas actividades de exigencia física ${ }^{11,12}$.

Por todo ello, junto a la escasez de estudios que evalúen la prevalencia de anemia en deportistas de alto nivel de Perú, se realizó este estudio que tuvo como objetivo describir la prevalencia de anemia ferropénica en profesionales del deporte y explorar la asociación de anemia ferropénica según sexo, edad y tipo de actividad deportiva, según el componente dinámico y estático del deporte en deportistas de alto nivel del Instituto Peruano del Deporte (IPD) en el año 2013.

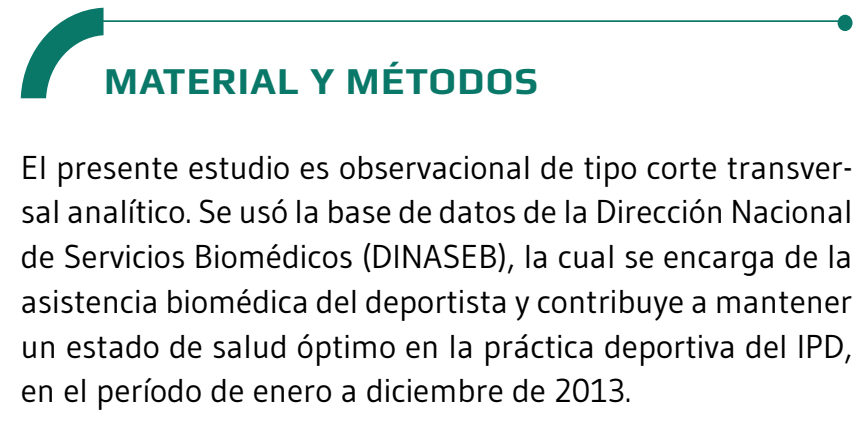

Muestra: Los profesionales del deporte $(n=633)$ incluidos en el estudio fueron aquellos deportistas a quienes en el año 2013 se les había realizado algún examen de laboratorio de rutina en la DINASEB del IPD. En este estudio se incluyeron los deportistas de ambos sexos de entre 18 a 35 años y se excluyeron a los deportistas de la selección de ajedrez y deportistas que residían en zonas con una altura mayor a 1000 m s. n. m. El diagrama de flujo puede consultarse en la Figura 1.

Figura 1. Diagrama de flujo de la muestra poblacional.

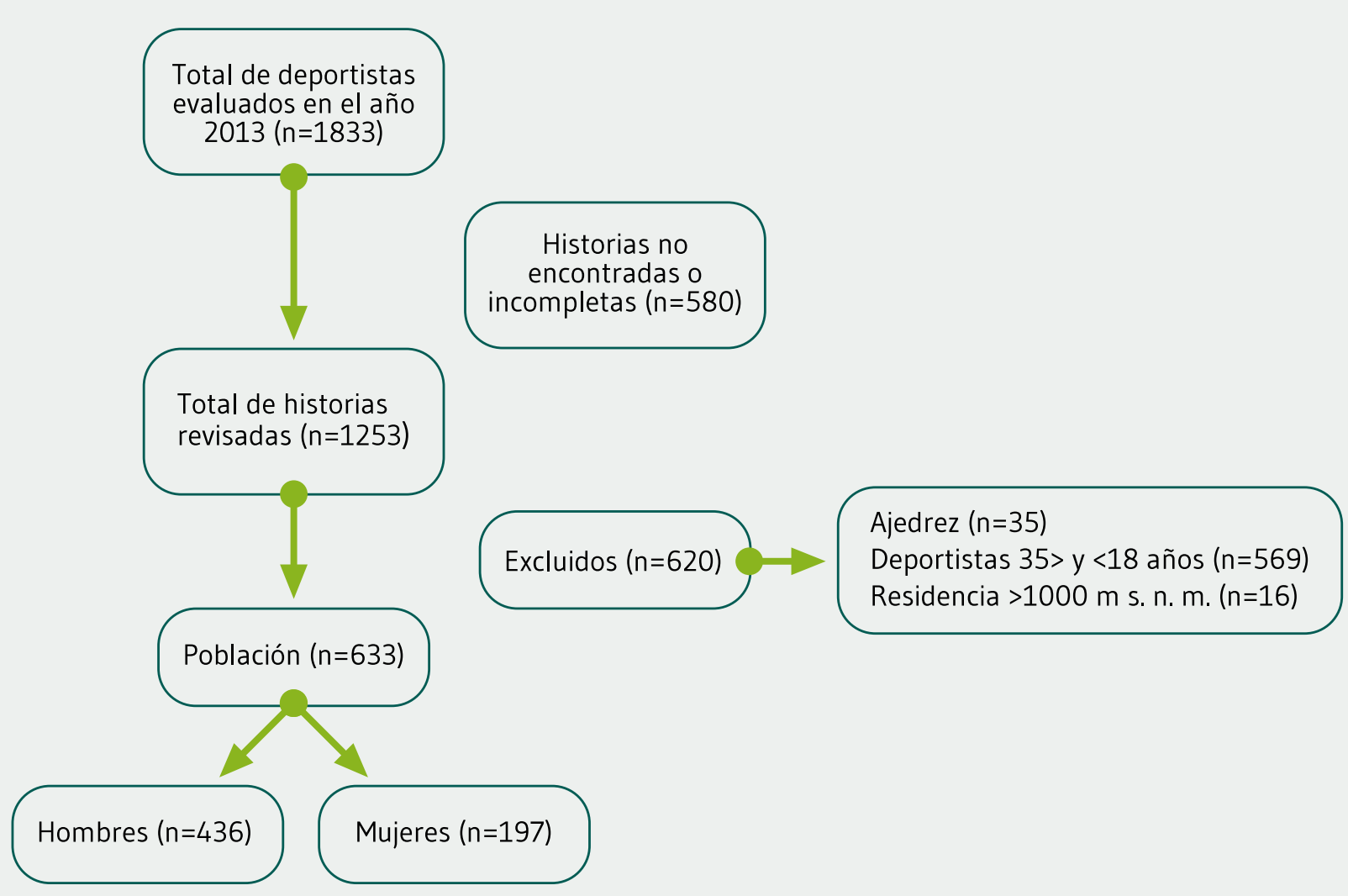


Instrumentos y procedimiento: Se recopiló información antropométrica e indicadores como edad, tiempo que llevan como seleccionado deportivo (edad deportiva), suplementación con hierro, índice de masa corporal, presión sistólica, hemoglobina, hematocrito, ferritina, colesterol y glucosa de los deportistas, recogidos de los análisis rutinarios de las historias clínicas. La información antropométrica incluía información del peso en kilogramos, talla en centímetros y presión arterial, medidos usando métodos estandarizados. También se obtuvo el índice de masa corporal como el peso en kilos divido por la talla en metros al cuadrado. Igualmen- te, se recogió información sociodemográfica (sexo, edad y residencia), la ingesta de suplementos de hierro e información sobre el tipo de deporte que practican, y la edad deportiva (que se clasificó según la mediana en menor de 7 años y mayor o igual de 7 años). Asimismo, con la información sobre la práctica deportiva se clasificaron los 45 deportes realizados en 9 categorías distintas, en donde se dividió a los deportistas según el nivel de intensidad del deporte (alto, mediano, bajo) en los ejercicios de categoría dinámica o estática durante el nivel competitivo usando el modelo de Task Force 8: Classification of Sports ${ }^{13}$ (Figura 2).

Figura 2. Clasificación de deportes según su componente dinámico y estático.

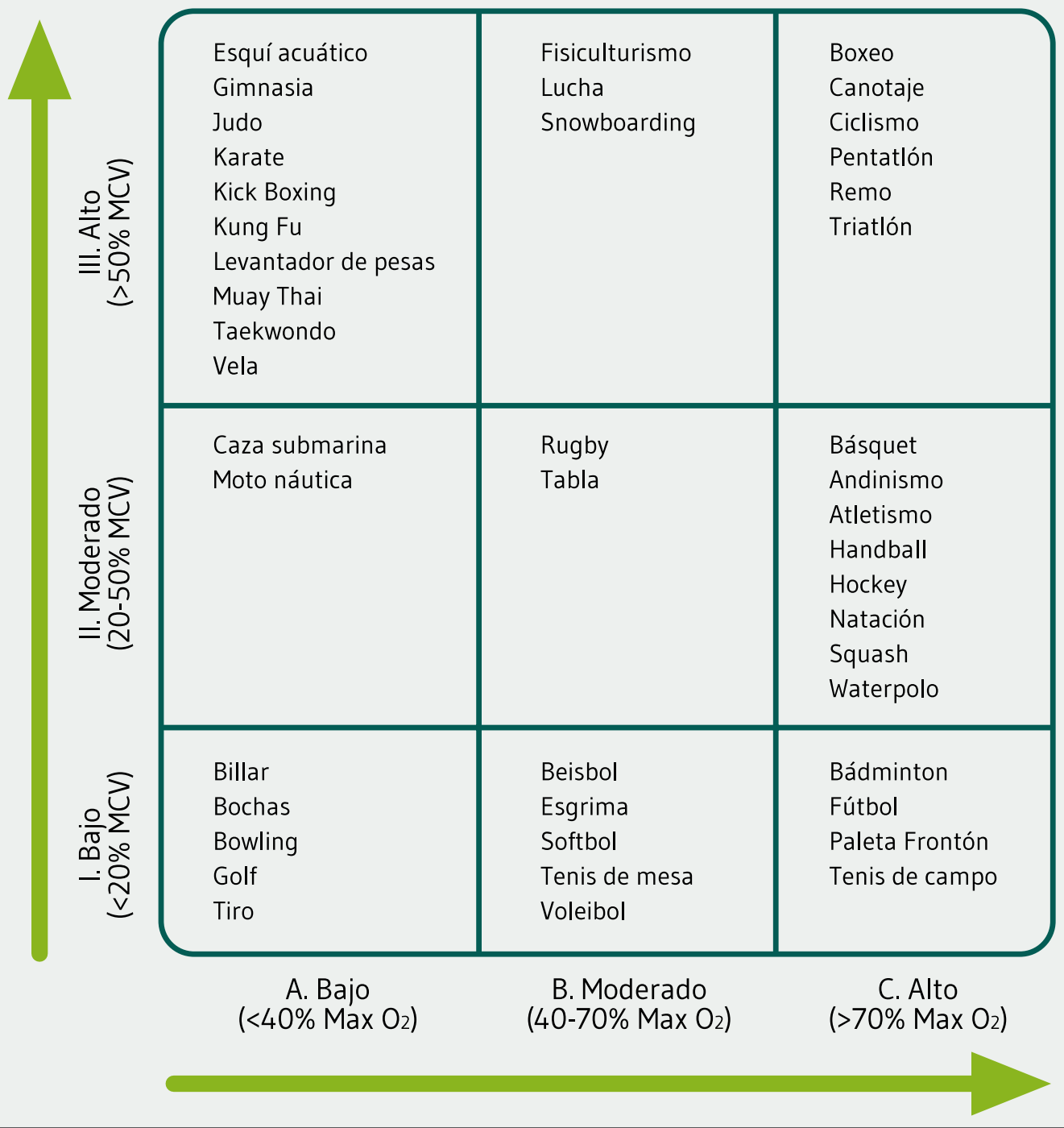

MVC: Máxima contracción voluntaria; Max $\mathbf{0}$ : Máxima capacidad de oxígeno.

*Figura adaptada de Task Force 8: Classification of Sports ${ }^{13}$. 
Análisis estadístico: Se realizó inicialmente un análisis descriptivo usando frecuencias para variables categóricas, y medidas de tendencia central y desviación para las variables numéricas. Se comprobó la normalidad de las variables numéricas utilizando el test de Kolmogorov Sminrov. Para comparar las diferencias entre la presencia y las variables numéricas se usó la prueba de T de Student o la prueba de Mann-Whitney dependiendo del estado de normalidad de las variables, y para comparar las diferencias en las variables cualitativas se usó la $\chi^{2}$.

Se calculó la razón de prevalencias crudas y ajustadas usando la regresión de Poisson con varianza robusta para evaluar la asociación entre género, edad y práctica deportiva; y anemia fue definida como presencia de anemia cuando la hemoglobina se encontraba en un rango $<13 \mathrm{mg} / \mathrm{dL}$ para hombres y $<12 \mathrm{mg} / \mathrm{dL}$ para mujeres ${ }^{1}$. El uso de la regresión de Poisson con varianza robusta en estudios transversales ha demostrado ser un método eficiente para el cálculo de razón de prevalencias ${ }^{14}$. Las variables incluidas en el modelo ajustado son aquellas que resultaron asociadas en el análisis crudo (valor $p<0,05$ ).

Aspectos éticos: Se obtuvo la autorización del Comité de Ética (CIE-UPC) de la Universidad Peruana de Ciencias
Aplicadas (UPC) y de la Dirección Nacional de Servicios Biomédicos para realizar el análisis de la base de datos secundaria disponible en DINASEB.

\section{RESULTADOS}

En el presente estudio se incluyen deportistas de ambos sexos con edad entre 18 y 35 años, de los cuales 436 fueron hombres, encontrándose que la mediana de edad del género masculino fue de 22,6 años $(19,9-26,7)$, mientras que en mujeres fue de 22 años $(19,8-25,6)$. Por otro lado, no se observó diferencia entre los promedios de hemoglobina haIlados en hombres $(14,8 \mathrm{~g} / \mathrm{dL})$ y mujeres $(13,0 \mathrm{~g} / \mathrm{dL})$. La prevalencia de anemia en la población de estudio fue de 17,5\% (IC95\%:14,8-20,7). La Tabla 1 muestra las características sociodemográficas, antropométricas, indicadores hematológicos y bioquímicos y de práctica deportiva de los participantes en el estudio para el total de la muestra y según tengan o no presencia de anemia. En la misma se puede observar que la población anémica posee niveles altos de triglicéridos y mayores niveles de ferritina en comparación a la población no anémica. Por último, tanto la presión sistólica como el

Tabla 1. Características generales de los deportistas seleccionados del IPD en el año 2013.

\begin{tabular}{|c|c|c|c|c|}
\hline Caracteristicas generales & Total & Anemia & Sin anemia & Valor $\mathbf{P}$ \\
\hline Mediana Edad en años & $633(22,2)$ & $111(22,0)$ & $522(22,3)$ & 0,74 \\
\hline Edad deportiva (DE)** & & & & 0,06 \\
\hline$<7$ años & $309(49,7)$ & $63(57,8)$ & $246(48,0)$ & \\
\hline >7 años & $313(50,3)$ & $46(42,2)$ & $267(52,1)$ & \\
\hline IMC ( $\left(\mathrm{kg} / \mathrm{m}^{2}\right)(\mathrm{DE})$ & $24,5(3,4)$ & $23,8(3,3)$ & $24,6(3,4)$ & 0,05 \\
\hline Presión sistólica (mmHg) (DE) & $109,3(10,5)$ & $108,1(10,4)$ & $109,5(10,3)$ & 0,18 \\
\hline Hemoglobina (g/dL) (DE) & $14,2(1,4)$ & $12,7(1,6)$ & $14,6(1,1)$ & 0,15 \\
\hline Hematocrito (\%) (RIQ) & $632(43,4)$ & $111(39,6)$ & $521(44,2)$ & 0,15 \\
\hline Ferritina $(\mu \mathrm{g} / \mathrm{L})(\mathrm{DE})^{*}$ & $112(124,0)$ & $87(115,6)$ & $25(262,6)$ & 0,15 \\
\hline Anemia (\%) & $633(100 \%)$ & $111(17,5 \%)$ & $522(82,5 \%)$ & \\
\hline Suplementación de hierro (\%)*** & $459(100 \%)$ & $82(19,2 \%)$ & $344(80,8 \%)$ & 0,88 \\
\hline Glucosa (mg/dL) (DE) & $82,5(7,4)$ & $82,3(7,3)$ & $82,5(7,4)$ & 0,83 \\
\hline Colesterol total (mg/dL) (DE) & $163,4(29,7)$ & $160,0(29,8)$ & $164,0(29,7)$ & 0,23 \\
\hline Triglicéridos (mg/dL) (RIQ) & $557(76,6)$ & $87(69,9)$ & $470(78,4)$ & 0,01 \\
\hline
\end{tabular}

DE: Desviación estándar; RIQ: Rango Interquartil.

*Los datos de ferritina comprenden 112 individuos (17,7\% de la población estudiada).

** Los datos comprenden un total de 622 individuos (98,3\% de la población estudiada).

*** Los datos comprenden un total de 459 participantes (72,5\% de la población estudiada). 
índice de masa corporal se ven ligeramente afectados por la anemia.

En la Tabla 2 se muestra la prevalencia de anemia según las 9 categorías en los 45 deportes estudiados. Finalmente, los deportes con menor prevalencia de anemia fueron béisbol, esgrima, softbol, tenis de mesa y voleibol (7,7\% de anemia; IC95\%:2,9-19,0) y aquellos con mayor prevalencia de anemia fueron piragüismo, ciclismo, pentatlón, triatlón, y remo (22,4\%; IC95\%:12,8-36,3).
Finalmente, las variables que se incluyeron en el modelo bivariado fueron género, edad deportiva y suplementación con hierro, las cuales fueron también incluidas en el modelo multivariado. En ambos análisis para razón de prevalencias ajustadas ( $\mathrm{RPa}$ ) se observó una asociación entre edad deportiva y anemia (RPa=0,6; IC95\%:0,4-0,9; $\mathrm{p}=0,01$ ) (Tabla 3). No se encontró asociación con las variables género ( $\mathrm{RPa}=1 ; \mathrm{IC} 95 \%: 0,6-1,4 ; \mathrm{p}=0,85)$ y suplementación con hierro (RPa=1,2; IC95\%:0,6-2,6; $\mathrm{p}=0,62$ ) (Tabla 3).

Tabla 2. Presencia de anemia según la clasificación de deporte en los deportistas seleccionados del IPD en el año 2013.

$n=633$

Alto componente estático - Alto componente dinámico

Alto componente estático - Bajo componente dinámico

Alto componente estático - Moderado componente dinámico

Bajo componente estático - Alto componente dinámico

Bajo componente estático - Bajo componente dinámico

Bajo componente estático - Moderado componente dinámico

Moderado componente estático - Alto componente dinámico

Moderado componente estático - Bajo componente dinámico

Moderado componente estático - Moderado componente dinámico

\begin{tabular}{|c|c|}
\hline \multicolumn{2}{|c|}{ Anemia } \\
\hline NO & Sí \\
\hline $38(77,5 \%)$ & $11(22,5 \%)$ \\
$142(81,1 \%)$ & $33(18,9 \%)$ \\
$28(77,8 \%)$ & $8(22,2 \%)$ \\
$54(84,4 \%)$ & $10(15,6 \%)$ \\
$39(81,3 \%)$ & $9(18,8 \%)$ \\
$48(92,3 \%)$ & $4(7,7 \%)$ \\
$89(81,7 \%)$ & $20(18,4 \%)$ \\
$10(90,9 \%)$ & $1(9,1 \%)$ \\
$74(83,1 \%)$ & $15(16,9 \%)$ \\
\hline
\end{tabular}

Tabla 3. Modelo Crudo y Ajustado de la Asociación de género y anemia en deportistas seleccionados del IPD en el año 2013.

\begin{tabular}{|l|c|c|c|c|c|c|c|} 
Variables & RP & IC95\% & Valor P & $\begin{array}{c}\text { RP } \\
\text { Ajustado }\end{array}$ & IC95\% & Valor P \\
\hline Género & 0,9 & $0,6-1,4$ & 0,73 & 1 & $0,6-1,4$ & 0,85 \\
Edad deportiva & 0,7 & $0,5-1$ & 0,06 & 0,6 & $0,4-0,9$ & 0,01 \\
Suplementación con hierro & 1,1 & $0,5-2,2$ & 0,88 & 1,2 & $0,6-2,6$ & 0,62 \\
\hline Tipo de deporte & & & & & & \\
\hline Alto componente estático - Alto componente dinámico & REF & & & REF & & \\
Alto componente estático - Bajo componente dinámico & 0,8 & $0,7-0,9$ & 0,57 & 0,2 & $0,7-0,9$ & 0,94 \\
Alto componente estático - Moderado componente dinámico & 0,7 & $0,6-0,9$ & 0,98 & 0,2 & $0,6-0,9$ & 0,69 \\
Bajo componente estático - Alto componente dinámico & 0,8 & $0,7-0,9$ & 0,36 & 0,2 & $0,7-0,9$ & 0,65 \\
Bajo componente estático - Bajo componente dinámico & 0,8 & $0,7-0,9$ & 0,65 & 0,2 & $0,7-0,9$ & 0,76 \\
Bajo componente estático - Moderado componente dinámico & 0,9 & $0,8-1$ & 0,05 & 0,8 & $0,8-1$ & 0,37 \\
Moderado componente estático - Alto componente dinámico & 0,8 & $0,7-0,9$ & 0,54 & 0,2 & $0,7-0,9$ & 0,62 \\
Moderado componente estático - Bajo componente dinámico & 0,9 & $0,5-1$ & 0,36 & 0,9 & $0,5-1$ & 0,60 \\
Moderado componente estático - Moderado componente dinámico & 0,8 & $0,7-0,9$ & 0,42 & 0,2 & $0,7-0,9$ & 0,85 \\
\hline
\end{tabular}

RP: Razón de prevalencias; IC95\%: Intervalo de Confianza del 95\%. 


\section{$\longrightarrow$ DISCUSIÓN}

El presente estudio obtuvo como principal resultado que no se encontró asociación entre la anemia y el género. Sin embargo, la prevalencia de anemia en la población de estudio fue de $17,5 \%$, (17,9\% del género masculino y $16,8 \%$ del género femenino). Por otro lado, los deportes con mayor predisposición a padecer anemia fueron piragüismo, ciclismo, pentatlón, triatlón y remo; y los deportes con menor prevalencia béisbol, esgrima, softbol, tenis de mesa y voleibol. Por último, no se encontró asociación entre edad deportiva y anemia. Estos resultados se acercan a lo mostrado en el estudio realizado en deportistas de Cali, Colombia ${ }^{15}$, y al de Dubnov y Constantini donde se presentó un $7 \%$ de anemia en el total de deportistas evaluados, de los cuales un $3 \%$ eran hombres y un $4 \%$ eran mujeres ${ }^{16}$. Sin embargo, estos resultados no coincidieron con los estudios realizados anteriormente, donde resultó que las mujeres presentaron una mayor prevalencia de anemia en el deporte ${ }^{17}$, donde se asocia una mayor predisposición a presentar anemia directamente a hemorragias fisiológicas generadas por la menstruación ${ }^{18}$. Del mismo modo, un estudio realizado a mujeres deportistas profesionales tuvo como resultado que el $60 \%$ presentó anemia, que, al compararlo con otros estudios en mujeres no deportistas, se llegó a la conclusión que las deportistas tenían una mayor prevalencia de anemia ${ }^{19}$, además de que se ha observado una mayor prevalencia de anemia en mujeres en la población en general ${ }^{20}$.

En Mar del Plata, Argentina, se identificó que un $25 \%$ de mujeres adultas padecían de anemia y la frecuencia de esta patología fue mayor en mujeres que en hombres $(29,5 \%$ y $25 \%)^{21}$. En Caracas, Venezuela, se muestra que en estudiantes preuniversitarios la anemia es mayor en mujeres llegando a un $28 \%{ }^{22}$. Por último, en adultos mayores de México la anemia afecta dos veces más al género femenino $(34,8 \%)$ que al masculino $(17 \%)^{23}$. Otro resultado del presente estudio refiere que, a mayor tiempo de practicar el deporte o edad deportiva, había un menor riesgo a presentar anemia. Sin embargo, otros estudios refieren que el deportista es normalmente sometido a largos días de entrenamiento deportivo, el cual genera un aumento del volumen plasmático y, como repercusión principal, una dilución de componentes sanguíneos como la hemoglobina ${ }^{24}$. Del mismo modo, se observa que la alta actividad física realizada genera una continua pérdida extra de hemoglobina por hemólisis, sudoración y en ciertos deportes por la alta cantidad de pisadas que se realiza en el entrenamiento y/o competencia ${ }^{25}$. Estos estudios muestran las variadas causas concomitantes acumulativas durante el tiempo de la práctica deportiva que, principalmente, actuarían como un factor sinérgico hacia la presencia de anemia en el deportista. Una investigación realizada a corredores de fondo y ciclistas mostró una deficiencia de hierro y ferritina ligada a su deporte; según el presente estudio, estos deportes son de alto componente dinámico y estático, y se identificó como el grupo con mayor prevalencia de anemia ${ }^{26}$. Por otro lado, se evaluó la composición corporal, nivel de hemoglobina y perfil nutricional en atletas de balonmano, obteniendo 14,29\% de anemia ${ }^{27}$, en comparación a nuestro estudio, donde se presentó un $18,4 \%$ de anemia en el mismo deporte.

En el presente estudio se evaluó una población de 18 a 35 años, debido a que se considera que la prevalencia de anemia aumenta directamente proporcional a la edad ${ }^{18}$, ligado a distintas complicaciones y causantes presentadas como ingesta insuficiente, deterioro en la capacidad de absorción ${ }^{28,29}$, afecciones inflamatorias y sangrados presentados en el tubo digestivo alto y bajo ${ }^{30,31}$. Asimismo, la clasificación de anemia utilizada fue basada en los puntos de corte establecidos por la OMS ( $<12 \mathrm{mg} / \mathrm{dL}$ en mujeres y $<13 \mathrm{mg} / \mathrm{dL}$ en hombres) ${ }^{1}$, los cuales son puntos de corte en la población general.

A pesar de no haber sido evaluado en este estudio, es conocido que los deportistas consumen una gran variedad de productos y suplementos entre los cuales se encuentra el hierro, sin especificar la dosis de suplementación farmacológica ${ }^{32}$. Se considera muy importante para estudios futuros registrar la dosificación de este micronutriente debido a que la alteración de las concentraciones de hierro por ingesta o aumento de las pérdidas del mismo, produce una situación de déficit en sus depósitos con disminución en las reservas orgánicas y finalmente en el surgimiento de anemia ${ }^{33}$.

Limitaciones y fortalezas: La investigación realizada tiene algunas limitaciones como todo estudio observacional. En primer lugar, el diseño del estudio realizado es transversal analítico; debido a ello, no se permite medir la causalidad entre género y el nivel de hemoglobina en estos deportistas. Otra limitación es que los datos recogidos no permiten describir qué tipo de anemia posee el deportista, ya que por ejemplo no se tenían los valores de ferritina en todos los participantes (para poder evaluar cuánta de la anemia es por deficiencia de hierro). El componente dietético es de gran importancia en el mundo deportivo, debido a que mejora la condición previa al esfuerzo físico y a su vez equilibra la pérdida electrolítica y energética durante el ejercicio ${ }^{34}$. El componente anteriormente nombrado se muestra también como una limitación, debido a que dentro del estudio no se evaluó la ingesta dietética del deportista.

Una de las fortalezas de la investigación es que en el Perú no se han encontrado estudios que evalúen la prevalencia 
de anemia en los deportistas seleccionados de alto nivel del IPD. Del mismo modo, la información que se analice será de importancia tanto para el área médica, la deportivo nutricional y para los propios deportistas que deseen informarse acerca de cómo influye el deporte que practican y los cuidados que deben observar para beneficio de su organismo. Por otro lado, este estudio tiene la fortaleza de usar la base de todos los deportistas calificados que están bajo el cuidado del ente rector nacional, lo que hace que la muestra sea representativa. Asimismo, estos resultados preliminares plantean la necesidad de realizar un estudio para evaluar las características de la anemia en deportistas.

\section{COnclusiones}

En conclusión, no se encontró asociación entre las variables anemia y género en los deportistas seleccionados del Instituto Peruano del Deporte durante el año 2013. Es importante mencionar que esta patología afecta a gran parte de los deportistas y que está asociada al tiempo de práctica deportiva. Por otro lado, se halló mayor prevalencia de anemia en los deportes de alto componente estático y dinámico tales como piragüismo, ciclismo, pentatlón, remo y triatlón. Finalmente, se propone el monitoreo regular de los niveles de hemoglobina y hierro en los deportistas para poder intervenir con un tratamiento pertinente y mantener un óptimo rendimiento.

\section{CONFLICTO DE INTERESES}

Los autores expresan que no hay conflictos de interés al redactar el manuscrito.

\section{REFERENCIAS}

(1) Organización Mundial de la Salud. Concentraciones de hemoglobina para diagnosticar la anemia y evaluar su gravedad. Ginebra, Suiza: Organización Mundial de la Salud; 2011.

(2) Farrús Palou M, Pérez Ocaña A, Mayer Pujadas MA, Piquer Gibert M, Mundet Tudurí X, Iglesias Rodal M. Anemias en atención primaria: etiología y características morfológicas. Aten Primaria. 2000; 25(4): 230-5.

(3) Instituto Nacional de Estadística e Informática. Perú: Encuesta Demográfica y de Salud Familiar - ENDES 2014. Lima, Perú: Instituto Nacional de Estadística e Informática; 2015.
(4) De Paz R, Hernández-Navarro F. Manejo, prevención y control de la anemia perniciosa. Nutr Hosp. 2005; 20(6): 433-5.

(5) Sacristán A, Estaca EG, Galinari HJ. Actividad física y salud en la mujer. Toko-Gin Pract. 2009; 68(2): 40-2.

(6) Urdampilleta A, Martínez-Sanz JM, Mielgo-Ayuso J. Anemia ferropénica en el deporte e intervenciones dietéticonutricionales preventivas. Rev Esp Nutr Hum Diet. 2013; 17(4): 155-64.

(7) Mouton G, Sluse FE, Bertrand A, Welter A, Cabay JL, Camus G. Iron status in runners of various running specialties. Arch Int Physiol Biochim. 1990; 98(1): 103-9.

(8) Dufaux B, Hoederath A, Hollmann W. Serumferritin bei Ruderinnen, Schwimmerinnen, Kurz, Mittel- und Langstrecklenläuferinnen. Dtsch Z Sportmed. 1988; 39(7): 260-4.

(9) Haymes EM, Spillman DM. Iron status of women distance runners, sprinters, and control women. Int J Sports Med. 1989; 10(6): 430-3.

(10) Pate RR. Sports Anemia: A Review of the Current Research Literature. Phys Sportsmed. 1983; 11(2): 115-31.

(11) Spodaryk K. Haematological and iron-related parameters of male endurance and strength trained athletes. Eur ] Appl Physiol Occup Physiol. 1993; 67(1): 66-70.

(12) Telford RD, Cunningham RB. Sex, sport, and body-size dependency of hematology in highly trained athletes. Med Sci Sports Exerc. 1991; 23(7): 788-94.

(13) Mitchell JH, Haskell W, Snell P, Van Camp SP. Task Force 8: classification of sports. J Am Coll Cardiol. 2005; 45(8): 1364-7.

(14) Coutinho LMS, Scazufca M, Menezes PR. Methods for estimating prevalence ratios in cross-sectional studies. Rev Saude Publica. 2008; 42(6): 992-8.

(15) Castillo M, Mora Al, Aldana L, Bermúdez MI, Piraneque A. Valoración del estado funcional del hierro en deportistas de alto rendimiento de las ligas de waterpolo y patinaje de Cali, Colombia. Nova. 2012; 10(17): 38-49.

(16) Dubnov G, Constantini NW. Prevalence of iron depletion and anemia in top-level basketball players. Int J Sport Nutr Exerc Metab. 2004; 14(1): 30-7.

(17) Legaz A. Atletismo español: Análisis básico de la pseudoanemia, anemia ferropénica y anemia megaloblástica. Rev int med cienc act fís deporte. 2000; 1(1): 65-83.

(18) McClung JP. Iron status and the female athlete. J Trace Elem Med Biol. 2012; 26(2-3): 124-6.

(19) DiehI DM, Lohman TG, Smith SC, Kertzer R. Effects of physical training and competition on the iron status of female field hockey players. Int J Sports Med. 1986; 7(5): 264-70.

(20) Gandra YR. La anemia ferropénica en América Latina y el Caribe. Bol Oficina Sanit Panam. 1970; 68(5): 375-87.

(21) Marín GH, Rivadulla P, Negro L, Gelemur M, Etchegoyen G, GIS. Estudio poblacional de prevalencia de anemia en población adulta de Buenos Aires, Argentina. Aten Primaria. 2008; 40(3): 133-8.

(22) Carías D, Cioccia AM, Gutiérrez M, Hevia P, Pérez A. Indicadores bioquímicos del estado nutricional de adolescentes preuniversitarios de Caracas. An Venez Nutr. 2009; 22(1): 12-9.

(23) Shamah-Levy T, Cuevas-Nasu L, Mundo-Rosas V, MoralesRuán C, Cervantes-Turrubiates L, Villalpando-Hernández S. Estado de salud y nutrición de los adultos mayores en México: resultados de una encuesta probabilística nacional. Salud Pública Méx. 2008; 50(5): 383-9. 
(24) Oscai LB, Williams BT, Hertig BA. Effect of exercise on blood volume. J Appl Physiol. 1968; 24(5): 622-4.

(25) Clement D, Asmundson R. Nutritional intake and hematological parameters in endurance runners. Phys Sportsmed. 1982; 10(3): 37-43.

(26) Nuviala RJ, Abos D, González E, Giner A. Valoración de las deficiencias de hierro en corredores de fondo y ciclistas. Arch Med Deporte. 1988; 5(17): 11-6.

(27) Guerra T, Knackfuss M, da Silveira C. Avaliação da composição corporal, nível de hemoglobina e perfil nutricional de atletas de handebol. Fitness Perform J. 2006; 5(5): 277-81.

(28) Salive ME, Cornoni-Huntley J, Guralnik JM, Phillips CL, Wallace RB, Ostfeld AM, et al. Anemia and hemoglobin levels in older persons: relationship with age, gender, and health status. J Am Geriatr Soc. 1992; 40(5): 489-96.

(29) Smith DL. Anemia in the elderly. Am Fam Physician. 2000; 62(7): 1565-72.
(30) Mukhopadhyay D, Mohanaruban K. Iron deficiency anaemia in older people: investigation, management and treatment. Age Ageing. 2002; 31(2): 87-91.

(31) Heikkinen A, Alaranta A, Helenius I, Vasankari T. Use of dietary supplements in Olympic athletes is decreasing: a follow-up study between 2002 and 2009. J Int Soc Sports Nutr. 2011; 8(1): 1.

(32) Newhouse IJ, Clement DB, Taunton JE, McKenzie DC. The effects of prelatent/latent iron deficiency on physical work capacity. Med Sci Sports Exerc. 1989; 21(3): 263-8.

(33) González-Gross M, Gutiérrez A, Mesa JL, Ruiz-Ruiz J, Castillo MJ. La nutrición en la práctica deportiva: Adaptación de la pirámide nutricional a las características de la dieta del deportista. Arch Latinoam Nutr. 2001; 51(4): 321-31. 